\title{
Hardware Design of SUAV Flight Control System Based on STM32F427
}

\author{
Tao Yang*, Liren Chai and Gang Wang \\ China Academy of Aerospace Aerodynamics, Beijing, 100074, China \\ *Corresponding author
}

\begin{abstract}
As an emerging high-tech device, the SUAV plays a more and more significant role in military and civilian fields because of its advantages such as low cost, high safety, flexible usability and easy maintenance. The flight control system is the core of the SUAV, which exerts a big influence on SUAV performance to a large extent. In order to meet the requirement of low power consumption, simple structure and high performance, this paper designs the hardware of SUAV flight control system based on STM32F427. The modular hardware design is introduced in detail, including the digital control circuit, the power circuit, the sensor circuit and the interface circuit. The flight control system designed in this paper is feasible, effective and reliable, fulfilling the design demand and improving the control and operation performance of the SUAV.
\end{abstract}

Keywords-SUAV; flight control; hardware design; STM32F427

\section{INTRODUCTION}

In recent times, study of the small unmanned aerial vehicle (SUAV) is attracting more and more attention as a result of its simple structure, easy maintenance, flexible take-off and landing, high cost-benefit ratio and great maneuvering performance[1,2]. SUAV is widely applied in different fields, accomplishing different tasks, to name a few, aerial photography, geographical mapping, remote sensing and telemetry, power line inspection, forest fire fighting, atmosphere monitoring, environmental protection, resource exploration, pesticide spraying and plant protection $[3,4]$. The glorious prospect arouses a large amount of manpower and financial investment in the study of all kinds of advanced SUAV technologies among various countries all over the world. With the utilization of new technologies, SUAV is becoming increasingly indispensable. The air-rang, flight height, flight duration, flight speed, ceiling, loading capacity, reliability and maintainability are the main performance indicators of SUAV, which depend largely on performance of the flight control system [5].

The flight control system is the top priority in the SUAV. The control system accepts the command from the ground station and acquires sensors output for data fusion. And then it sends the control command to different actuators according to the corresponding control laws. The flight control system conducts navigation, control, communication and other core missions during the SUAV flight [6]. It is of big research value when it comes to autonomous flight, route planning, airborne equipment management, cooperative control of multiple vehicles, and security administration of prohibited area. For higher flight quality, the flight control system has to respond rapidly and work efficiently, while low power consumption, small size and light weight is also required. Stable and effective hardware design of the flight control system is the basis of all these. Under this background, the hardware is designed with two STM32F427 as its core. One STM32F427 executes the control algorithm and digital communication, captures PWM input signals, and outputs PWM driving signals. In the meantime, the other one communicates with all kinds of sensors.

\section{WORKING PRINCIPLES OF THE UAV FLIGHT CONTROL SYSTEM}

During the SUAV flight missions, the flight control system downloads waypoint information and instructions of the ground station via data radio at first. And then the flight parameters are obtained according output of different sensors, thus calculating the current SUAV attitude and position. According to the waypoints set in advance, the flight control system figures out the actuator control value, with it the control surface rotating correspondingly [7]. As shown in Figure I, the data communication system conducts data communication between the ground station and the flight control system, ensuring correct transmission of the command and waypoints information. After analysis of the sensor data, the flight control system sends the position command to the actuator, thereby changing the flight attitude. What's more, with the help of data radio, the flight control system sends back the flight status and the airborne equipment status to the ground station for display and control, in order to inform the manipulator of the real-time flight attitude and position, thus ensuring the flight security and stability in case of emergency with the stringent control exerted in time by the manipulator. To summarize, function of the flight control system can be divided into two parts. On one hand, the flight control system keeps the SUAV flight safe and stable with preset rules, and changes the attitude and waypoints after receiving new instructions from the ground station. One the other hand, it manages airborne equipment, including flight parameters acquisition and status parameters storage as well as control and monitoring of the airborne equipment. 


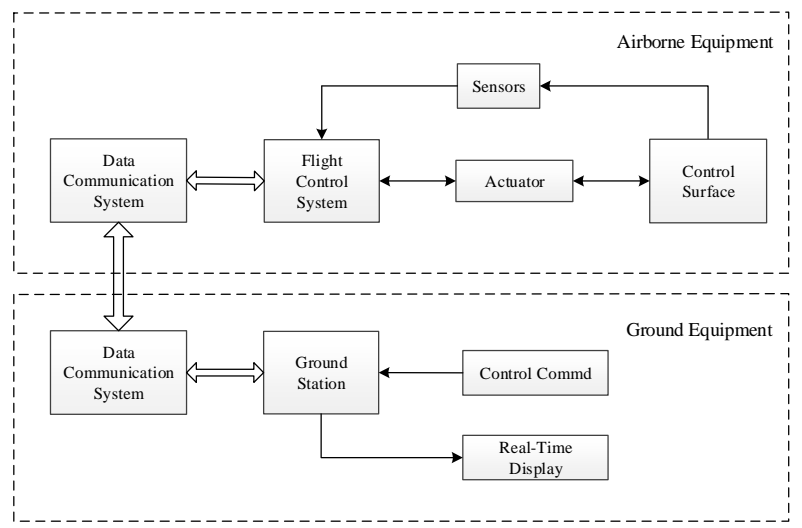

FIGURE I. BLOCK DIAGRAM OF THE FLIGHT CONTRL SYSTEM

\section{THE HARDWARE DESIGN}

Due to the complex hardware design of the flight control system. It is introduced in the form of modular design with the following 4 sections: the digital control circuit, the power circuit, the sensor circuit and the interface circuit. There are two control chips STM32F427 in the digital control circuit. One cooperates with the other one to conduct digital control. The power circuit offers power supply for the whole hardware. The sensor circuit is made of various sensors which provides the attitude and position reference. The interface circuit contains the communication interface and the PWM and capture interface.

\section{A. The Digital Control Circuit}

The digital control circuit contains two control chips STM32F427. The STM32F427 device is based on the high-performance ARM Cortex-M4 32-bit RISC core operating at a frequency of up to $180 \mathrm{MHz}$. The Cortex-M4 core features a floating point unit, and it implements a memory protection unit. It incorporates high-speed embedded memories with Flash memory up to 2 Mbyte, SRAM up to 256 Kbytes, and backup SRAM up to 4 Kbytes. There are various enhanced I/Os and peripherals as well as standard and advanced communication interfaces. Thanks to its high computing speed, large Flash and SRAM memroy, powerful peripherals, and rich communication interfaces, STM32F427 is one of the perfect choices for SUAV flight control.

As shown in Figure II, the STM32F427 in the left is the control core of the flight system with the control algorithm performed in it. It captures the PWM input signals to obtain the actuator throttle command, and it generates the corresponding PWM driving signals for the actuator. It reserves the UART, CAN and SBUS communication interfaces. As for data uploading and downloading, a FRAM chip with memory up to 256KB stores the control parameters and preset waypoints information; a 8GB EMMC chip records the airborne flight data online in real time; all the data mentioned above can be downloaded from the flight control system by a USB interface for offline data analysis and flight replay. A UART interface links the left STM32F427 and the right one together. The STM32F427 in the right acquires information from all kinds of sensors, calculates the flight attitude and position, and reports back to the left control chip simultaneously. The 3-Axis digital compass module combines 3-axis magneto-resistive sensors and 3-axis MEMS accelerometers; The 3D AHRS/VRU/IMU module outputs 3D orientation, 3D rate of turn, 3D accelerations, and 3D magnetic field; The altimeter provides a precise digital 24 bit pressure and temperature value; The GNSS module can receive up to three GNSS systems (GPS/Galileo) together with BeiDou or GLONASS) concurrently. Also there is a FRAM chip recording the configuration parameters of the various sensors.

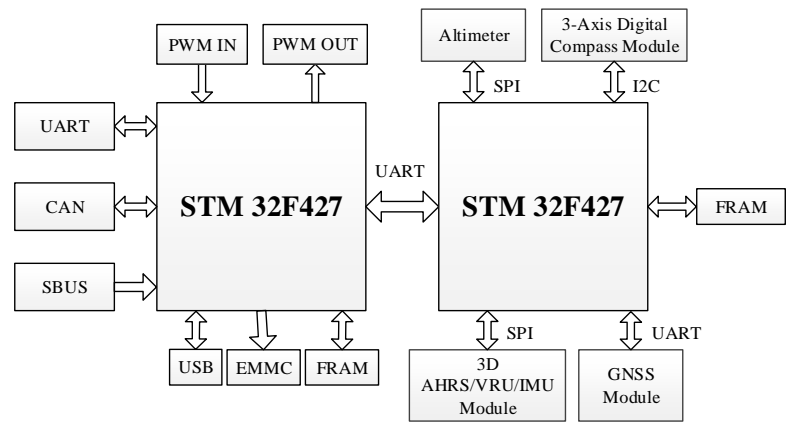

FIGURE II. BLOCK DIAGRAM OF THE DIGITAL CONTROL CIRCUIT

\section{B. The Power Circuit}

The system power input can be from batteries or the DC power supply, with a wide range input from $9 \mathrm{~V}$ to $36 \mathrm{~V}$. As shown in Figure III, the complete 8A switch mode DC/DC power supply LTM4613 transforms the power input into $+6 \mathrm{~V}$ with ultralow noise as the power source of actuator PWM driving output. At the same time, the electromagnetic compatible (EMC) DC/DC buck converter LTM8033 transforms the power input into $+5 \mathrm{~V}$, with output current up to $3 \mathrm{~A} .+5 \mathrm{~V}$ is the power source of USB interface. The low-dropout linear regulator TPS74091 transforms $+5 \mathrm{~V}$ into $+3.3 \mathrm{~V}$, a multiple power source for the control core STM32F427, the altimeter, the GNSS module, the 3D AHRS/VRU/IMU module, the 3-Axis digital compass module, the UART and CAN communication circuit, and the FRAM and EMMC chip.

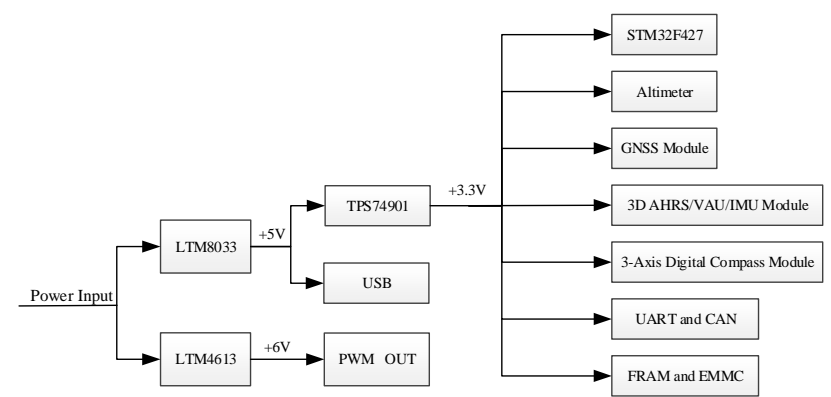

FIGURE III. SYSTEM POWER SUPPLY

\section{The Sensor Circuit}

1) The $3 D$ AHRS/VRU/IMU module

As shown in Figure IV, the 3D AHRS/VRU/IMU module selected is MTi-3-AHRS-8A7G6. It is available as an inertial measurement unit (IMU), vertical reference unit (VRU) or attitude and heading reference system (AHRS). This module is fully functional with different self-contained components, 
rendering it easy to design. And only a few hardware components are needed. Signals are fully processed onboard, requiring very little resources from the control chip STM32F427 over SPI interface. The host can read out the data by customized frequency and output format. Its roll/pitch accuracy is $1.0^{\circ} \mathrm{RMS}$, and its yaw accuracy is $2^{\circ}$ RMS under dynamic conditions, which is excellent for control and navigation of SUAV.

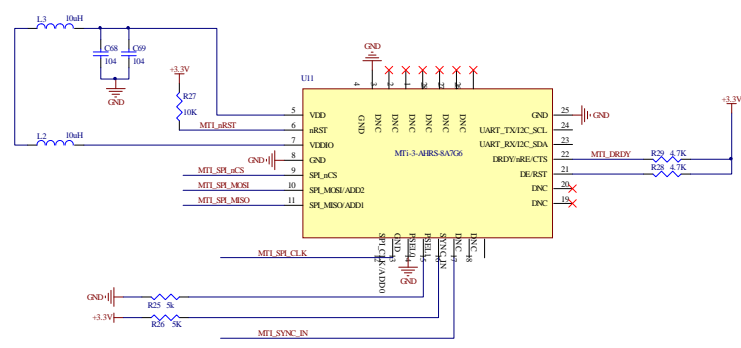

FIGURE IV. THE 3D AHRS/VRU/IMU MODULE CIRCUIT

\section{2) The altimeter}

As shown in Figure V, the altimeter sensor selected is MS5803. It is a high-resolution 24 bit altimeter sensor, communicating with STM32F427 via SPI interface. Its altitude resolution can be up to $20 \mathrm{~cm}$. There are different operation modes, allowing the user to optimize for conversion speed and current consumption. The communication protocol is simple, without the need of programming internal registers in the device.

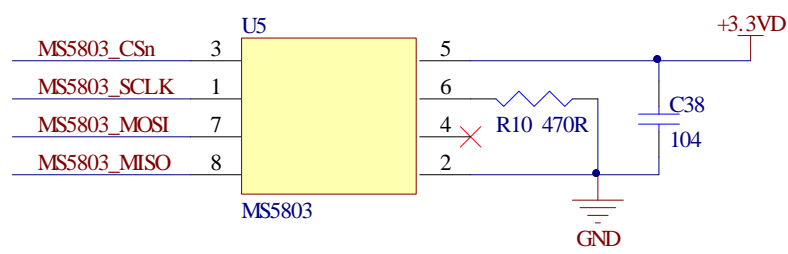

FIGURE V. THE ALTIMETER CIRCUIT

\section{3) The GNSS module}

As shown in Figure VI, the GNSS module sensor selected is MAX-M8. MAX-M8 communicates with the flight control system via UART interface. It recognizes multiple constellations simultaneously and provides outstanding positioning accuracy in scenarios with urban canyon or weak signals. It offers high performance even at low power consumption levels. It provides best performance for passive and active antennas designs of the SUAV.

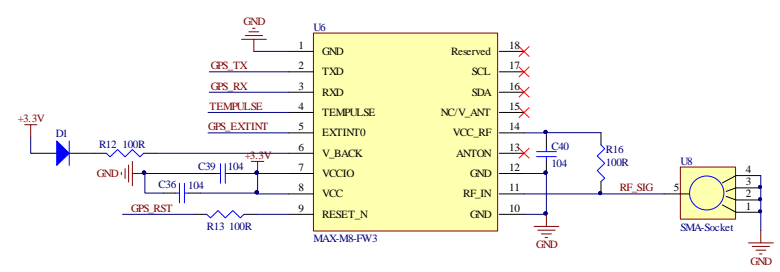

FIGURE IV. THE GNSS MODULE CIRCUIT

4) The 3-Axis digital compass module

As shown in Figure VII, the 3-Axis digital compass selected is HMC6343. Through I2C interface, MC6343, a fully integrated compass module combining 3-axis magneto-resistive sensors and 3-axis MEMS accelerometers with required analog and digital support circuits, transmits the heading computation results to the flight control system. The sensor features precision sensitivity and linearity, as well as solid-state construction with very low cross-axis sensitivity designed to measure both direction and magnitude of Earth's magnetic fields.

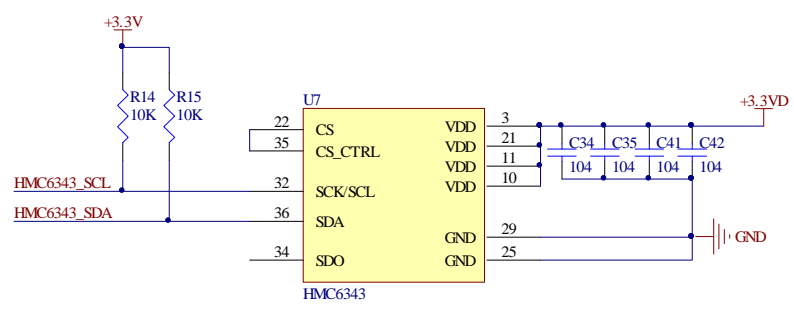

FIGURE VII. THE 3-AXIS DIGITAL COMPASS MODULE CIRCUIT

\section{The Interface Circuit}

The interface circuit is made up of two kinds of interfaces, namely, communication interface and the PWM and capture interface. The communication interfaces are UART, CAN and SBUS interfaces. The UART conducts the communication between the flight control system and the data radio as well as the data and parameter transmission between the two control chips STM32F427. The SBUS interface connects with a SBUS receiver, exclusive for Futaba SBUS protocol. The rest communication interfaces are backups for future extension. The control system captures 6 channels of PWM input signals and outputs 16 channels of PWM driving signals. The electric level of PWM input signals is $+5 \mathrm{~V}$, which has to be transformed into $+3.3 \mathrm{~V}$ to meet the requirement of STM32F427 I/O level. For more powerful driving ability, the PWM output signal has the electric level of $+6 \mathrm{~V}$.

\section{CONCLUSION}

As the advance of science and technology, the SUAV is playing a more and more significant role in modern world, no matter whether it comes to the military field or the civilian fields. As the brain of SUAV, the flight control system must perform properly and stably without any mistakes. The hardware of the flight control system based on STM32F427is designed in this paper, with advantages of small size and light weight. After several ground tests in our research group, this flight control hardware proves to be feasible and reliable, and it works efficiently while the power consumption low, thus fulfilling the design demand and improving the control and operation performance of the SUAV.

\section{REFERENCES}

[1] Yang Lei, The Desgin of Small UAV Flight Control System Based on STM32, Harbin: Northeast Forestry University, 2016.

[2] Zhou Wenxia, Research on UAV Flight Control Computer Platform Based on ARM, Nanjing: Nanjing University of Aeronautics and Astronautics, 2008.

[3] Hu Yong, Ding Wanshan, Design of the Hardware Platform for a UAV Flight Control System Based on DSP, Metrology and Measurement Technology. 5 (2004) 35-38. 
[4] Wang Wei, Design of UAV Flight Control Computer Based on DSP, Nanjing: Nanjing University of Aeronautics and Astronautics, 2009.

[5] Yu Xiaozhou, Zhou Fengqi, Huanghe, Hardware Platform for the UAVs based on the SOPC Technology, Fire Control and Command Control. 12 (2006) 48-50.

[6] Wang Bin, Design of a UAV Flight Control Computer System, Nanjing: Nanjing University of Aeronautics and Astronautics, 2007.

[7] Liang Shuang, Design and Implement of Hardware and Attitude Reference System for the Flight Control System of Small UAV, Chongqing: Chongqing University, 2016. 